\title{
Multi-Port Fiber Optic Beam Splitters for Space Division Multiplexed (SDM) Systems
}

\author{
ANDREW WOOD*, YONGMIN JUNG AND DAVID J. RICHARDSON \\ Optoelectronic Research Centre, University of Southampton, Southampton, SO17 1BJ, UK \\ *asjw1g12@soton.ac.uk
}

\begin{abstract}
We incorporate two major types of SDM fibers (i.e. few mode fibers and multicore fibers) in a micro-optic collimator assembly and realize fully integrated multiport SDM fiber components (e.g. $1 \times 2$ and $2 \times 2$ beam splitters). The device fabrication procedure for SDM fibers is similar to that for a standard single mode fiber, but more accurate fiber alignment (e.g. lateral positioning for few mode fiber components and rotational alignment for multicore fiber components) is required to achieve both low mode (core) dependent loss and/or low inter-modal (inter-core) crosstalk. A few exemplary multiport SDM components are fabricated with low excess loss (1-2 dB) and low mode (core) dependent loss $(<1 \mathrm{~dB})$.
\end{abstract}

C 2019 Optical Society of America under the terms of the OSA Open Access Publishing Agreement

\section{Introduction}

With core/backbone optical telecommunication networks edging ever closer to their fundamental Shannon capacity limit [1], there has been growing research interest in space division multiplexed (SDM) transmission $[2,3]$ to explore this final degree of freedom available for signal multiplexing. Over the past 10 years, various types of SDM fiber (e.g. few mode fibers and multicore fibers) have been introduced and investigated as a means to provide multiple spatial channels in a single optical fiber. As a result, substantial research progress has been made and many remarkable data transmission records have been set. For example, $>100$ spatial channel fiber transmission [4], $>40$ spatial channel amplification [5, 6] and $>10$ petabit/s total fiber capacity [7]. Despite this, many key components are still not available in an SDM compatible form, multiport SDM fiber beam splitters (e.g. $1 \times 2$ or $2 \times 2$ splitters) are one such component. Beam splitters are devices that take an input beam(s) and split it into two components according to the device's power coupling ratio, allowing for passive branching of signals within a network. Current methods for creating such devices are based on fused biconical taper (FBT) and planar lightwave circuit technology, both of which can deliver commercial single mode fiber devices with highly controllable splitting ratios; however, no such products are currently available for both few mode fibers (FMFs) and multicore fibers (MCFs). Recently, a FMF coupler has been experimentally demonstrated using the FBT approach [8]; however due to the $\mathrm{LP}_{11}$ mode having a larger mode field diameter than the fundamental $\mathrm{LP}_{01}$ mode, the $\mathrm{LP}_{11}$ mode starts to couple to the output fiber before the $\mathrm{LP}_{01}$ mode, meaning that it is very difficult to achieve a $3 \mathrm{~dB}$ coupling ratio. One of the easiest solutions, should someone need an SDM fiber beam splitter, is to use a bulk free space optics set-up (e.g. bulk beam splitter and a pair of lenses) [9]; however, this method is cumbersome and, whilst usable in the laboratory, is not appropriate for large scale deployment in an SDM network.

Very recently, we have demonstrated a compact approach to SDM fiber components using micro-optic collimator technology [10]. In principle, any type of SDM fiber can be integrated in to such an assembly and a high-density 32-core isolator has previously been successfully demonstrated with reasonable optical loss $(<2 \mathrm{~dB}$ for all cores $)$. All of our previous devices demonstrations were focused on 2-port fiber optic components having a single input and a single output. However, in this paper we further extend the approach to multi-port (3 or 4-port) 
SDM fiber components. As a demonstration, both FMF and MCF optical beam splitters are fabricated with low excess loss.

\section{Fabrication of the SDM fiber beam splitters}

Figure 1(a) shows a schematic of our 3-port fiber optic beam splitter, allowing light propogating on a single input SDM fiber to be split into two identical SDM fiber outputs. The device is based on micro-optic collimator assemblies and a partially reflective mirror (chosen to have a $50 / 50$ power splitting ratio in our experiment (although any desired splitting ratio can in principle be chosen) is used to reflect half the light down the reflected fiber port (i.e. port1 $\rightarrow$ port2), whilst the other half gets transmitted to a collimator that couples the remaining light to the transmitted output fiber (i.e. port1 $\rightarrow$ port3).

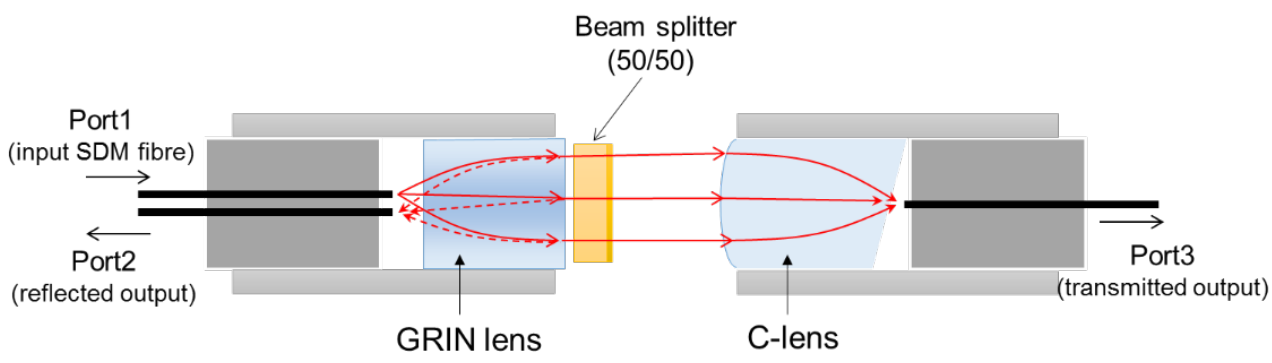

(a)

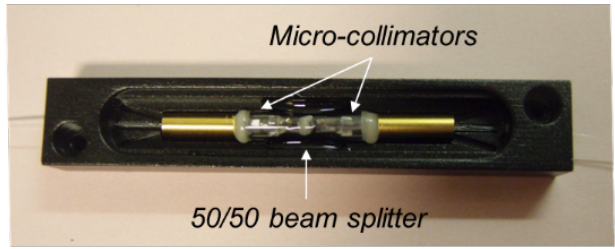

(b)

Fig. 1. (a) 3-port fiber optic beam splitter using SDM fibers and (b) a fully packaged SDM fiber beam splitter. Package dimensions $=12($ width $) \times 9$ (height $) \times 70 \mathrm{~mm}($ length $)$.

Fabrication of the device starts by creating a dual fiber collimator (left collimator assembly in Fig. 1a). This involves inserting two SDM fibers into a dual-hole glass ferrule (one hole housing the input with the other the reflected output fiber) and collimating the light from an input fiber using a graded-index (GRIN) lens. One crucial aspect of getting the light to reflect back down the output fiber is that the position of both these fibers are co-located in the same ferrule. To facilitate this, two SDM fibers were freely inserted into a dual-hole glass ferrule until they meet the surface of the GRIN lens. After which their positions were fixed, allowing the collimation process between the two fibers to be done at the same time and thus making our fiber alignment process considerably easier. Once the dual fiber collimator assembly is completed, the next step is to align the partially reflective mirror chip (dimension $=1.4 \times 1.4 \mathrm{~mm}$ ) on the top of the GRIN lens (outer diameter $=1.8 \mathrm{~mm}$ ) such that it reflects light back down the output fiber (port1 $\rightarrow$ port2). In our experiment, an optic tweezer was used to hold the edges of the mirror (i.e. to act as a mechanical clamp for preventing the mirror from moving during the epoxy curing process) and which was then mounted on a 5-axis stage platform for accurate optical alignment. The best way to realize accurate optical alignment is to first move the mirror far away from the collimator and to roughly align the mirror using a visible light source (e.g. a HeNe laser) so that the reflected beam is returned back down the output fiber. Once rough optical alignment has been obtained, a near infrared laser source and power meter can be used to achieve more accurate alignment. Here, the mirror can be moved closer to the GRIN lens and 
can be fixed in close proximity using a very high viscosity epoxy. The high viscosity prevents the epoxy from leaking in-between the mirror and the lens. Further to this, the whole setup can be mechanically held in place to prevent the mirror from moving whilst the epoxy is settling, since any movement at this stage can result in a loss of power at the output end. The epoxy is slowly cured using a low power UV lamp to further prevent any movement in the mirror.

Next, a single fiber collimator is created using a micro-optic C-lens (effective focal length $=2.98 \mathrm{~mm}$, outer diameter $=1.8 \mathrm{~mm}$ ) as shown on the right-hand side of the fiber collimator in Fig. 1(a). This fabrication process is nearly the same as for the dual fiber collimator, but only a single fiber is needed this time for light collimation. Once the single fiber collimator has been made, the two fiber collimator assemblies can be mounted on individual 5-axis translation stages which are then actively tuned to achieve the maximum coupling efficiency from input fiber to transmitted output fiber (port1 $\rightarrow$ port3). An external glass tube is then attached to add extra protection to the assembly and is fixed in place using a low-shrinkage epoxy. As shown in Fig. 1(b), the device can be packaged in a very compact format - our package dimensions are $12 \times 9 \times 70 \mathrm{~mm}$ (width $\times$ height $\times$ length respectively). Naturally, further manufacturing steps need to be taken according to the fiber type used: in the case of few mode fiber (FMF), light in the $\mathrm{LP}_{11}$ mode of the input fiber was extinguished (by careful bending) during the alignment process leaving just the $\mathrm{LP}_{01}$ mode, which gives the best center-to-center alignment for the system; in the multicore fiber (MCF) case, an additional rotation stage was added so that that the cores of the output MCF can be rotationally aligned to match those of the input MCF.

\section{3. $1 \times 2$ beam splitter for few mode fibers}

The FMF used in this demonstration was a graded-index two mode-group fiber (OFS Denmark), with a cladding diameter of $125 \mu \mathrm{m}$, a fiber attenuation of $<0.22 \mathrm{~dB} / \mathrm{km}$, and a differential group delay (DGD) of $+80 \mathrm{ps} / \mathrm{km}$. A binary phase plate based free-space setup was used to excite the individual spatial modes of the FMF and to characterize the mode dependent optical performance of the device. The excess loss was calculated by subtracting the output port power (in $\mathrm{dBm}$ ) from a reference power obtained from an identical fiber as used in the device (again in $\mathrm{dBm}$ ) and accounting for the $3 \mathrm{~dB}$ loss of the 50/50 mirror). The power is approximately split equally between the two output ports, with an excess loss of $\sim 1.4 \mathrm{~dB}$ for the $\mathrm{LP}_{01}$ mode and $\sim 2 \mathrm{~dB}$ for the $\mathrm{LP}_{11}$ mode. The higher loss of the $\mathrm{LP}_{11}$ mode can be accounted for by the comparatively larger mode field diameter and beam divergence when compared to the LP 01 mode. However, note that all micro-optics lenses (e.g. GRIN lens and C-lens) used in our experiment are originally designed for conventional single mode fiber operation and there is significant scope for further improvement in terms of MDL of the device by optimizing these micro-optic components. However, this is beyond the scope of the current paper. 

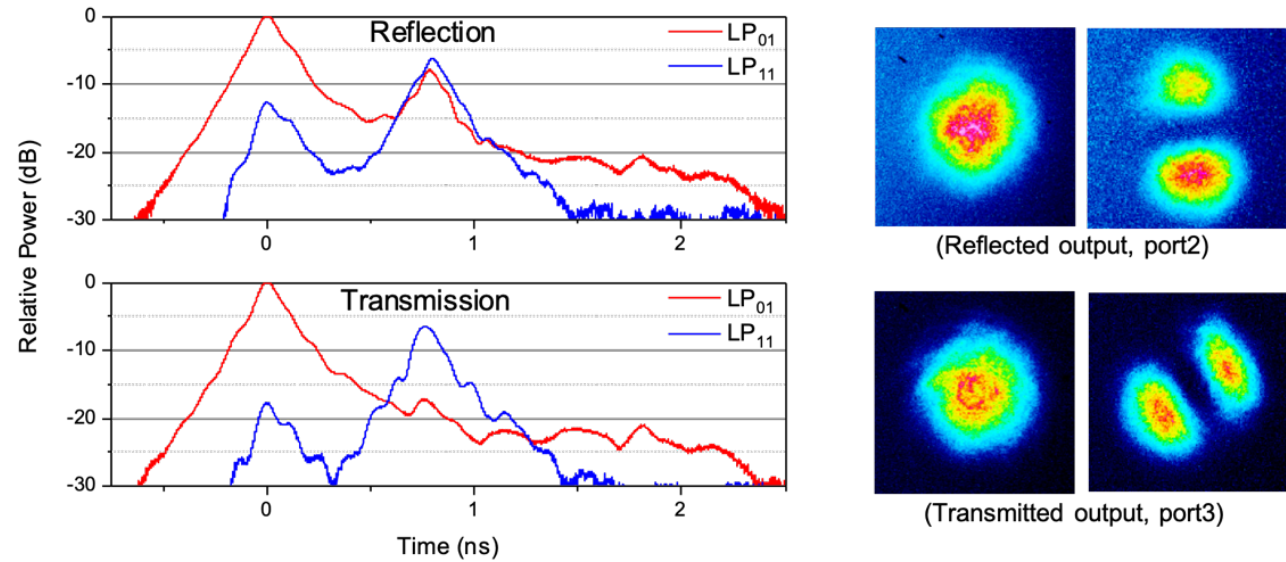

(Reflected output, port2)

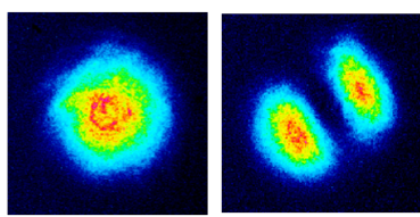

(Transmitted output, port3)

Fig. 2 Time of-flight measurement data along with the output modes for both reflected and transmitted outputs.

In order to investigate the modal purity of the fabricated device, a time-of-flight measurement (i.e. impulse response) was taken at both output ends. This involves launching pulses from a pulsed laser down a long piece of FMF (10 km in this case) placed after the chosen splitter port, with an oscilloscope recording the temporal output trace. Due to the two spatial modes having a different DGD through the fiber, there will be distinct peaks associated with each individual mode, allowing a measurement of the modal crosstalk as shown in Fig. 2 (left). It is clear that the transmission output has less cross-talk than the reflected output, exhibiting an $\mathrm{LP}_{11}$ mode suppression of $11 \mathrm{~dB}$, compared to the reflected output's $6 \mathrm{~dB}$. The higher crosstalk in the reflected port might be due to the tight alignment tolerance for tilting of the mirror coupled with epoxy shrinkage during the curing process. The fiber's modal images at both reflected and transmitted outputs are also measured using a CCD camera and relatively clean mode profiles are confirmed as shown in Fig. 2 (right). To characterize the polarization dependency of the fabricated FMF beam splitter, a polarization controller was placed at the beginning of the system and the power difference between the maximum and minimum was taken as the input polarization was varied. The results show that both the transmitted and reflected ports are similar to each other, with a power difference of $0.12 \mathrm{~dB}$ on average. This indicates a very low polarization dependent device loss for both $\mathrm{LP}_{01}$ and $\mathrm{LP}_{11}$ modes.

\section{4. $2 \times 2$ beam splitter for few mode fibers}

Moreover, the $1 \times 2$ FMF beam splitter described above can be further extended to realize a $2 \times 2$ FMF beam splitter by replacing the single fiber collimator on the right-hand side of Fig. 1(a) with a dual fiber collimator. This results in a four port devices consisting of two input ports and two output ports as shown in Fig. 3(a). Light from port 1 goes to port 2 and port 4, while light from port 2 goes to ports 1 and 3 . The mode dependent insertion loss was measured and is shown in the table at the bottom of Fig. 3(a). Note that this includes a $3 \mathrm{~dB}$ insertion loss from the $50 / 50$ beam splitter and the average excess loss was $\sim 0.5 \mathrm{~dB}$ for the $\mathrm{LP}_{01}$ and $\sim 1.5 \mathrm{~dB}$ for the $\mathrm{LP}_{11}$ modes, respectively. Similar to the previous observation from a $1 \times 2 \mathrm{FMF}$ beam splitter, the fabricated $2 \times 2$ FMF beam splitter also exhibits slightly higher insertion loss for the $\mathrm{LP}_{11}$ mode $\left(\sim 1 \mathrm{~dB}\right.$ more) compared to $\mathrm{LP}_{01}$ mode. Figure 3(b) shows the time-of-flight measurements of the fabricated $2 \times 2$ FMF beam splitter. A modal purity of more than $9.5 \mathrm{~dB}$ for all spatial modes was readily achieved for both the reflection and transmission ports. Note that the modal purity of the devices is very sensitive to the fiber center-to-center alignment and improved device performance could be achieved with further improved control of the optical alignment and packaging processes. 


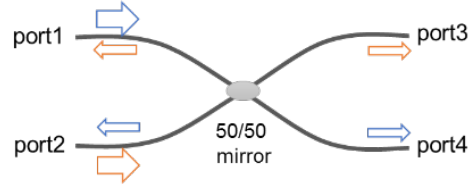

\begin{tabular}{|c|c|c|c|c|c|}
\hline \multirow{2}{*}{$\begin{array}{l}\text { Insertion } \\
\text { loss (dB) }\end{array}$} & \multicolumn{4}{|c|}{ OUT } \\
\cline { 3 - 6 } & Port1 & Port2 & Port3 & Port4 \\
\hline \multirow{3}{*}{ IN } & \multirow{2}{*}{ Port1 } & & $-3.4(\mathrm{LP} 01)$ & & $-3.7(\mathrm{LP} 01)$ \\
\cline { 3 - 6 } & & $-4.3(\mathrm{LP} 11)$ & & $-5.0(\mathrm{LP} 11)$ \\
\cline { 2 - 6 } & Port2 & $-3.4(\mathrm{LP} 01)$ & & $-3.7(\mathrm{LP} 01)$ & \\
& $-4.2(\mathrm{LP} 11)$ & & $-4.3(\mathrm{LP} 11)$ & \\
\hline
\end{tabular}

(a)

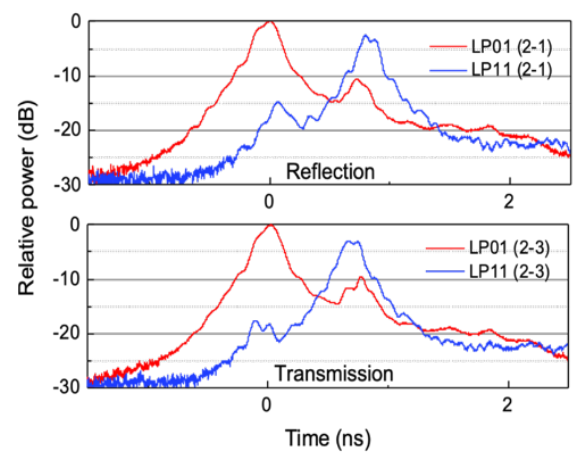

(b)

Fig. 3 (a) Schematic of $2 \times 2$ FMF beam splitter and the measured insertion loss. (b) Time-of-flight measurement results of the beam splitter.

\section{5. $1 \times 2$ beam splitter for multicore fibers}

MCF components present different challenges compared to FMF components, namely the rotational alignment of both the input and output fibers has to be matched to achieve low insertion loss for all guided cores. In our experiment, a four-core fiber is used as an exemplary MCF and a microscope image of the fiber cross section is shown in Fig. 4(a). The MCF has four graded-index single-mode cores which are trench-assisted to reduce the inter-core crosstalk. The fiber has a $10.8 \mu \mathrm{m}$ core dimeter, $125 \mu \mathrm{m}$ cladding diameter and $36 \mu \mathrm{m}$ core pitch distance. The measured inter-core crosstalk is lower than $-54 \mathrm{~dB} / \mathrm{km}$ and the overall fiber loss is $0.6-0.7 \mathrm{~dB} / \mathrm{km}$. In order to simultaneously excite all the cores of the MCF seen in Fig. 3 (a) the single mode fiber (SMF) coming from the laser was spliced onto a core-less fiber (i.e. pure silica fiber without any core), which in turn was spliced onto the MCF. The core-less fiber allows the light exiting the SMF to 'spread out', eventually illuminating all four cores of the fiber. The overall excess loss of the device was obtained in much the same way as the FMF case and a loss of $1.2 \mathrm{~dB}$ was determined for both sides. When dealing with a MCF, it is more important to calculate the excess loss of the individual cores as opposed to the device as a whole. To do this, a single mode fiber probe scanning method was used in the near field of the MCF to obtain the power distribution across the fiber cores. The probe was scanned over an area of $100 \mu \mathrm{m}$ by $100 \mu \mathrm{m}$ and sampled on a 100 by 100 step grid. Reference power intensities were first taken using a $1 \mathrm{~km}$ length of the same MCF, which can be seen in Fig. 3 (b), with the core numbers suitably labelled. After this, the fabricated $1 \times 2 \mathrm{MCF}$ splitter was spliced to the end of the fiber to obtain the excess loss for each of the cores. The average excess loss for the reflected and transmitted output was $1.9 \mathrm{~dB}$ and $2.2 \mathrm{~dB}$ respectively and the core-to-core variation was less than $1 \mathrm{~dB}$. 
a)

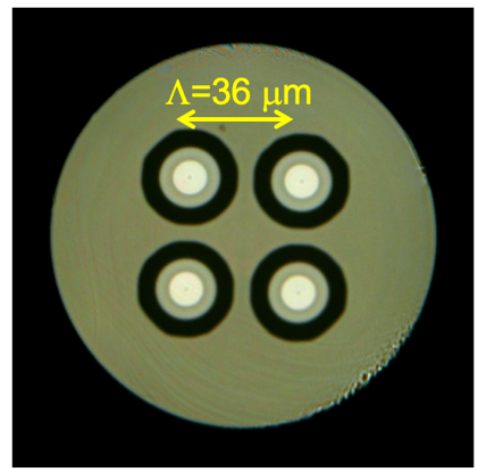

b)

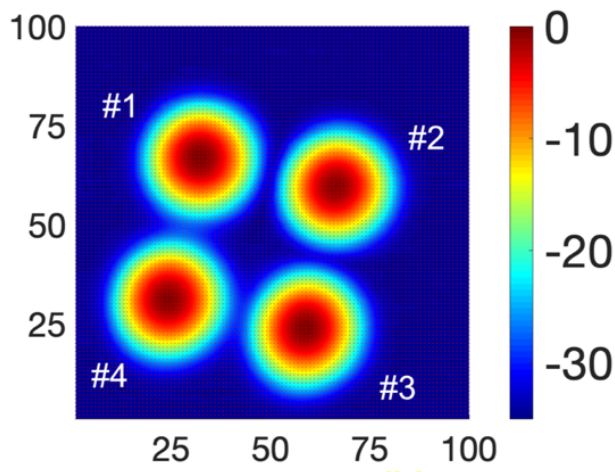

Fig. 4. (a) Cross sectional view of the 4-core fiber and (b) reference power intensity of the core without the core numberngs. Scale is in $\mathrm{dB}$.

Crosstalk is another important consideration in multicore fiber devices. To obtain the crosstalk values, a SMF was placed close to the input end of the MCF such that the light from the laser only illuminates one of the cores. From this, light in any of the other cores at the output end of the fiber can be attributed as crosstalk within the device. Therefore, a crosstalk matrix can be obtained by scanning the output end of the fiber. Figure 5 shows the inter-core crosstalk for all the cores of the transmitted output. The average crosstalk value was $-38 \mathrm{~dB}$ for the transmitted output and $-39 \mathrm{~dB}$ for the reflected output. The highest crosstalk value across both outputs was $-32 \mathrm{~dB}$.
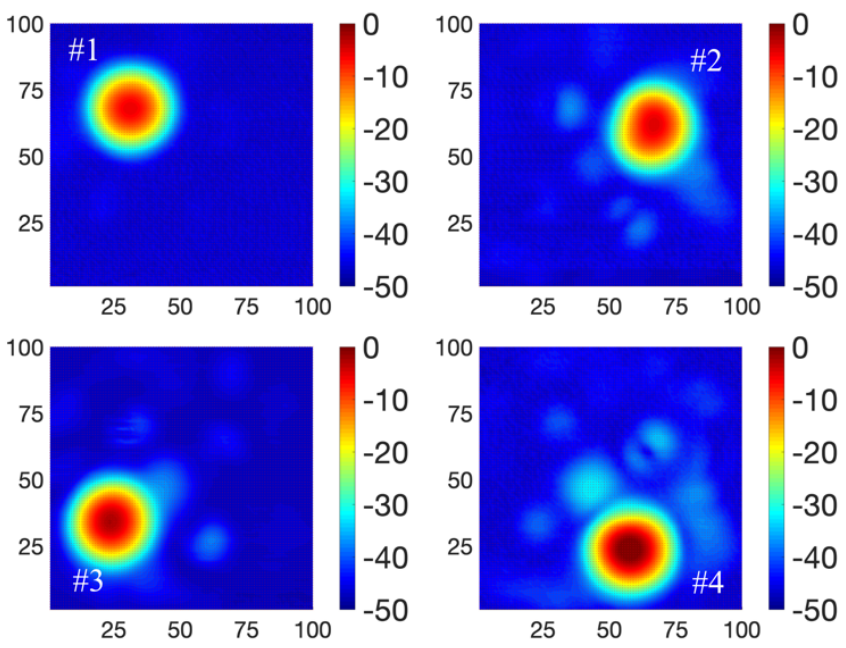

Fig. 5. Inter-core crosstalk of the 4 -core $1 \times 2$ beam splitter. Scale is in $\mathrm{dB}$.

\section{Conclusion}

Using micro-optic collimator technology we have successfully fabricated both 3-port and 4port beam splitters for both few mode fibers and multicore fibers, the first multiport fiber components for SDM transmission. All fabricated devices show very good optical performances, particularly for the FMF beam splitters with an excess loss of $0.5-1.6 \mathrm{~dB}$ and a mode dependent loss of $\sim 1 \mathrm{~dB}$ while the MCF beam splitter shows $\sim 1.2 \mathrm{~dB}$ averaged excess 
loss across all cores. Crosstalk better than $-32 \mathrm{~dB}$ was achieved. These preliminary results show that the design and fabrication methods used can be adopted to create compact, reliable passive devices. There is also considerable further scope for device optimization and it is hoped that they will find use for beam splitting/monitoring functions in future large scale, SDM networks.

\section{References}

1. R. Essiambre, G. Kramer, P. J. Winzer, G. J. Foschini and B. Goebel, "Capacity Limits of Optical Fiber Networks," J. Lightwave Technol. 28(4), 662-701(2010).

2. D. J. Richardson, J. M. Fini, and L. E. Nelson, "Space-division multiplexing in optical fibres," Nat. Photonics 7, 354-362 (2013).

3. R. Ryf et al., "Mode-Division Multiplexing Over 96 km of Few-Mode Fiber Using Coherent MIMO Processing," J. Lightwave Technol. 30(4), 521-531(2012).

4. T. Sakamoto et al., "120 Spatial Channel Few-Mode Multi-Core Fibre with Relative Core Multiplicity Factor Exceeding 100," ECOC'18, paper We3E.5. Rome, Sep. 2018.

5. Y. Jung, M. Wada, T. Sakamoto, S. Jain, I. A. Davidson, P. Barua, J. R. Hayes, S. Alam, K. Nakajima, and D. J. Richardson, "High Spatial Density 6-Mode 7-Core Multicore L-Band Fiber Amplifier," OFC'19, paper Th1B.7, San Diego, 3-7 March 2019.

6. S. Jain, C. Castro, Y. Jung, J. Hayes, R. Sandoghchi, T. Mizuno, Y. Sasaki, Y. Amma, Y. Miyamoto, M. Bohn, K. Pulverer, M. Nooruzzaman, T. Morioka, S. Alam, and D. J. Richardson, "32-core erbium/ytterbium-doped multicore fiber amplifier for next generation space-division multiplexed transmission system," Opt. Express 25, 32887 (2017).

7. D. Soma, Y. Wakayama, S. Beppu, S. Sumita, T. Tsuritani, T. Hayashi, T. Nagashima, M. Suzuki, M. Yoshida, K. Kasai, M. Nakawawa, H. Takahashi, K. Igarashi, I. Morita, M. Suzuki, "10.16 Peta b/s SDM/WDM transmission over 6 mode 19 core fiber across the C+L band," J. Lightwave Technol. 36, 1362-1369 (2018).

8. Y. Jung, R. Chen, R. Ismaeel, G. Brambilla, S. -U. Alam, I. P. Giles, and D. J. Richardson, "Dual mode fused optical fiber couplers suitable for mode division multiplexed transmission," Opt. Express 21, 24326-24331 (2013)

9. V. A. J. M. Sleiffer, Y. Jung, M. Kuschnerov, S. U. Alam, D. J. Richardson, L. Grüner-Nielsen, Y. Sun, and H. de Waardt, "Optical chopper-based re-circulating loop for few-mode fiber transmission," Opt. Lett. 39, 1181$1184(2014)$

10. Y. Jung, A. Wood, S. Jain, Y. Sasaki, S. Alam, and D. J. Richardson, "Fully integrated optical isolators for space division multiplexed (SDM) transmission," APL Photonics 4, 022801 (2019).

\section{Acknowledgements}

This work was supported by the EPSRC National Hub in High Value Photonic Manufacturing (EP/N00762X/1), the EPSRC funded "Airguide Photonics" Programme Grant (EP/P030181/1) and the EPSRC Impact Acceleration Account Award (EP/R511766/1).

Data contained in this paper are openly available from the University of Southampton repository at http://doi.org/10.5258/SOTON/XXXXX. 